\title{
Review of two-dimensional materials for photocatalytic water splitting from a theoretical perspective
}

\author{
Yunguo Li, ${ }^{a, b *}$ Yan-Ling Li, ${ }^{a}{ }^{\ddagger}$ Baisheng Sa, ${ }^{c}$ and Rajeev Ahuja ${ }^{d}$
}

\author{
Accepted 14 Nov 2016 \\ First published online 21 Nov 2016 \\ DOI: $10.1039 / C 6 C Y 02178 F$
}

Two-dimensional (2D) materials have shown extraordinary performances as photocatalysts compared to their bulk counterparts. $A b$ initio simulations that made great contribution to the understanding and design of novel $2 \mathrm{D}$ photocatalysts, not only show efficiency and reliability in searching new structures, but also provide a reliable, efficient, and economic way for screening the photocatalytic property space. In this review, we summarize the recent developments in the field of photocatalytic water splitting using 2D materials from a theoretical perspective. We address that $A b$ initio based simulations can fast screen the potential spaces of photocatalytic properties with the accuracy comparable to experiments, by investigating the effects of various physical/chemical perturbations. This, at last, will produce the enhanced photocatalytic activities of 2D materials, and promote the development of photocatalysis.

\section{Introduction}

Nowadays, the demand for clean energy has never been greater given the increasing human population and the ever growing energy consumption. Clean and sustainable energies are the cornerstone of the ecotopian society in the future. Jules Verne once wrote in his famous book that: "...water will one day be employed as fuel, that hydrogen and oxygen which constitute it, used singly or together, will furnish an inexhaustible source of heat and light...". He must be glad as this came true in 1972 when Fujishima and Honda ${ }^{1}$ split water into $\mathrm{H}_{2}$ and $\mathrm{O}_{2}$ using a photoelectrochemical (PEC) method. $\mathrm{H}_{2}$, when sourced in a clean and efficient manner, is the most ideal solution to the future energy demand.

In the PEC method, a bias must be applied on the electrocatalyst. In 1979 , the $\mathrm{TiO}_{2}$ powder was found to be able to split water without a bias voltage under exposure of sunlight with a sacrificial reagent ${ }^{2,3}$. This revealed the most promising and economical way to produce $\mathrm{H}_{2}$. Afterwards, tremendous progresses have been made on the photocatalytic process. More and more studies were reported in the last decade as seen in the number of publications (see Fig. 1). Timely reviews have summarized the revolutionary studies on semicon-

a School of Physics and Electronic Engineering, Jiangsu Normal University, Xuzhou, People's Republic of China. E-mail: yunguo@kth.se (Yunguo Li); ylli@jsnu.edu.cn (Yan-Ling Li).

${ }^{b}$ Crystallography and Mineral Physics, Department of Earth Sciences, University College London, Gower Street, London WC1E 6BT, United Kingdom.

${ }^{c}$ College of Materials Science and Engineering, Fuzhou University, People's Republic of China

d Condensed Matter Theory Group, Department of Physics and Astronomy, Uppsala University, Box 516, Uppsala 75120, Sweden. ductor photocatalysis ${ }^{4-8}$. Photocatalytic water splitting relies on the photogenerated electron-hole (e-h) pairs with high redox capacity. Linsebigler et al. ${ }^{4}$ classified photocatalytic water splitting into two classes depending on where the initial excitation occurs. The first process referred to as the sensitized photoreaction observes the initial photoexcitation in the catalyst substrate and then the excited electron is transferred into a ground-state molecule. The second process called the catalyzed photoreaction observes the initial photoexcitation in an adsorbate molecule which then interacts with the groundstate catalyst. Here, we restrict ourselves to the first case in this review. In this case, the electronic structure of the semiconductor plays an important role in water splitting. Electron in the valence band (VB) of semiconductors can be excited into the conduction band (CB) when absorbing a photon with energy no less than the bandgap energy, and subsequently a hole is also produced in the VB. Because of the presence of bandgap, the photogenerated e-h pairs can migrate to the surface of a semiconductor before recombination, where they can exchange charge and energy with adsorbed species $\left(\mathrm{OH}^{-}, \mathrm{O}_{2}\right.$, $\mathrm{O}^{-2}$,etc. $)^{9}$. New species $\left(\mathrm{OH}, \mathrm{O}_{2}^{-}\right.$, etc.) with high redox capacities will be created, which are the reagents for $\mathrm{H}_{2}$ and $\mathrm{O}_{2}$ production. The e-h pairs can also interact directly with these reagents ${ }^{9}$. The overall photocatalytic water splitting process refers to the reaction:

$$
\begin{array}{r}
2 \mathrm{H}_{2} \mathrm{O}(\mathrm{l}) \stackrel{\text { photon energy }>1.23 \mathrm{eV}}{=} \mathrm{O}_{2}(\mathrm{~g})+2 \mathrm{H}_{2}(\mathrm{~g}) \\
\Delta \mathrm{E}^{0}=1.23 \mathrm{~V}
\end{array}
$$

where $\Delta \mathrm{E}^{0}$ is the equilibrium potential at standard conditions. The PEC path to water splitting involves the oxidation and 
reduction half-cell reactions:

$$
\text { Oxidation : } \begin{aligned}
2 \mathrm{H}_{2} \mathrm{O}(\mathrm{l})= & \mathrm{O}_{2}(\mathrm{~g})+4 \mathrm{H}^{+}(\mathrm{aq})+4 \mathrm{e}^{-} \\
& \Delta \mathrm{E}^{0}=1.23 \mathrm{~V} \text { vs. SHE }
\end{aligned}
$$

and

$$
\begin{array}{r}
\text { Reduction : } 4 \mathrm{H}^{+}(\mathrm{aq})+4 \mathrm{e}^{-}=2 \mathrm{H}_{2}(\mathrm{~g}) \\
\Delta \mathrm{E}^{0}=0.00 \mathrm{~V} \text { vs. SHE }
\end{array}
$$

The changes of the redox potentials under different conditions can be expressed by the Nernst equation:

$$
E^{e}=\mathrm{E}^{0}-\frac{\mathrm{R} T}{\mathrm{zF}} \ln \frac{\text { oxidized }}{\text { reduced }}
$$

where, $E^{e}$ is the potential of the electrode, $\mathrm{R}$ is the universal gas constant, $\mathrm{F}$ is the Faraday constant, $z$ is the number of moles of electrons transferred in the reaction, and $T$ is the temperature. Therefore, for qualified photocatalysis, the potential level of the $\mathrm{CB}$ should be more negative than the $\mathrm{H}^{+} / \mathrm{H}_{2}$ potential to let the reduction (Eq. 3) proceed. While the VB energy level should be more positive than the $\mathrm{OH}^{-} / \mathrm{O}_{2}$ potential to proceed the oxidation (Eq. 2). Thus, the criteria of bandgap for photocatalysis corresponds to the minimum potential difference, and that is $1.23 \mathrm{eV}^{9}$ as shown in Fig. 2 . Many semiconductors can meet up with the bandgap criteria but the band alignment fails to be consent with the redox levels of water. However, as indicated in Equation 4, the concentration of $\mathrm{H}^{+}$( $\mathrm{pH}$ value) can change the reaction potential, and therefore may enable some semiconductors to be eligible for overall photocatalytic water splitting.

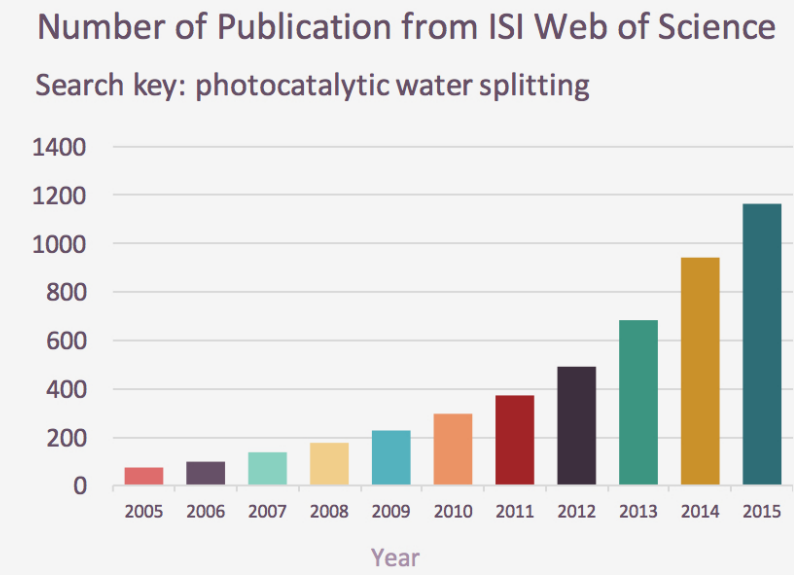

Fig. 1 Number of publications on photocatalytic water splitting per year over the last decade.

Apart from the basic criteria, efficient photocatalysis for water splitting must fulfill a Goldilocks principle: (a) High photon-harvesting efficiency in visible light region; (b) Good mobility of charge carriers; (c) Low recombination rate of e$\mathrm{h}$ pairs; (d) Abundant active sites and fast exchange rate of charge and energy with other species. Since the discovery of $\mathrm{TiO}_{2}$ as photocatalyst, great efforts have been devoted to the design of new efficient photocatalyst. Some semiconductors (e.g., $\mathrm{ZnO}, \mathrm{CdSe}, \mathrm{CdS}, \mathrm{WO}_{3}$, and $\mathrm{SrTiO}_{3}$, etc. $)^{9,10}$ were successively discovered to have good photocatalytic capacities. Techniques like doping, straining, defect engineering, applying electric field and heterogeneous structure construction are useful for further improvement of the photocatalytic properties. Nano-sized semiconductors with exotic properties have been shown to exhibit enhanced photocatalytic properties ${ }^{7}$. It is easily perceived that nano-sized materials have more reactive surface area, especially the one-dimensional (1D) and two-dimensional materials (2D) with dangling bonds. Compared to 1D photocatalysis, 2D photocatalysis have bigger surface/volume ratio, and are easier to be handled in the production and recycle processes. Improvement of the photocatalytic properties of these low-dimensional materials are also attributed to the improved e-h separation, fast mobility of charge carriers and reduced recombination rate.
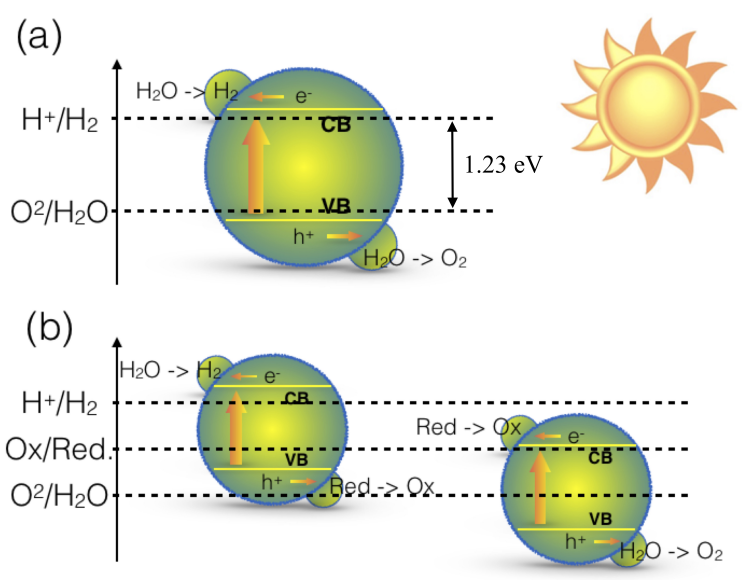

Fig. 2 Schematic representation of the photocatalytic water splitting process: (a) overall water splitting into $\mathrm{H}_{2}$ and $\mathrm{O}_{2}$ by photocatalyst and (b) Z-scheme photocatalysis for overall water splitting. Ox and Red designate the oxidized species and reduced species, respectively.

Since the discovery of Graphene ${ }^{11}$, many 2D materials were successfully fabricated with unique electronic structures and potential applications in photocatalytic water splitting. The boom of $2 \mathrm{D}$ materials that progressively promoted the development of photocatalysis are also benefited from the development of ab initio based modeling. The grand structural searching is becoming practical and more and more efficient, and new 2D materials are being discovered within shorter 
and shorter times. Accurate descriptions of the photocatalytic properties of 2D materials can also be achieved by the ab initio based modeling. Besides, simulations are more efficient and economic than experiments in searching conditions where can exert the best photocatalytic performances of $2 \mathrm{D}$ materials. It is timely and necessary to have a review on the techniques for engineering $2 \mathrm{D}$ photocatalysis. Therefore, we organize such a review and focus on screening 2D materials for photocatalytic water splitting. In this review, we discuss the discovery of 2D photocatalysis from a theoretical perspective and the accuracy of simulations in Section 2. In Section 3, the techniques for property engineering are summarized and discussed in the context of experimental and theoretical results. Challenges and perspectives are outlined in Section 4.

\section{Discovery of 2D photocatalysis}

\subsection{Prediction of $2 D$ structures}

For decades scientists have theorized about Graphene but never identified it until the enlightening work by Geim and Novoselov ${ }^{11}$. They found that the layers of graphite can be mechanically exfoliated while the single layers kept intact. Now, more complex single-layer structures can also be isolated by the emerged advanced methods. However, the success is always based on the weak binding between the layers in the bulk materials.

The most practiced methods for 2D materials' synthesis include the micromechanical cleavage, liquid phase exfoliation, photoexfoliation, anodic bonding, physical vapor deposition (PVD), chemical vapor deposition (CVD), Molecular Beam Epitaxy (MBE), sol-gel method, atomic layer deposition (ALD), and so on. These methods can be classified into the physical methods and the chemical methods, and some of them are suitable for mass production. These methods either ask for highly skilled researchers or expensive equipment. Besides, people do not know exactly which materials are 2D candidates until they succeed in fabricating them.

On the other hand, theory is able to predict 2D materials and has played an important role in the rapid growth of the 2D materials' world. The theoretical study on 2D materials started early in $1940 \mathrm{~s}^{12}$. Many 2D materials were first predicted by theory before they can be experimentally synthesized or observed, ike Graphyne ${ }^{13-15}$, Silicene ${ }^{16,17}$, and Borophene ${ }^{18}$.

The $a b$ initio crystal structure prediction needs an accurate theoretical description of many-body systems, which is still one of the biggest challenges in solid state sciences though significant progresses have been made. For a system with many electrons and nuclei, the electronic and nuclei systems can be treated separately following the Born-Oppenheimer approximation. The Hatree-Fock (HF) method ${ }^{19}$ can solve the electronic Schrödinger equation (SE) explicitly by expanding the wavefunction in the Slater determinant. But the correlation of electrons is totally neglected (except for the exchange correlation arising from pairs of electrons with the same spin), and the bond energies are significantly underestimated. Post HF methods with expanded determinants involving excited orbitals have been developed to include the electron correlation corrections, but it is at extreme expense level (the computation scales like $N^{5}$ and higher ${ }^{19,20}$ ) and only restricted to simple systems with small unit cells. Alternatively, Hohenberg, Kohn and Sham proved that the complicated $N$-electron wavefunction is not necessary and the total electron density $\rho$ can determine exactly and completely all the (ground-state) properties $^{21,22}$. Their approach, widely known as the density functional theory (DFT), reduces the complexity and maps the many-body SE onto a series of Kohn-Sham (KS) singleparticle Schrödinger equations:

$$
\begin{array}{r}
\left\{-\frac{\hbar^{2}}{2 m_{e}} \nabla_{j}^{2}+\int \sum_{j^{\prime} \neq j} \psi_{j^{\prime}}^{\mathrm{KS}}\left(\mathbf{r}^{\prime}\right) \frac{q^{2}}{\left|\mathbf{r}-\mathbf{r}^{\prime}\right|} \psi_{j^{\prime}}^{\mathrm{KS}}\left(\mathbf{r}^{\prime}\right) d \mathbf{r}^{\prime}+V_{e n}(\mathbf{r})\right. \\
\left.+V_{x c}(\mathbf{r})\right\} \psi_{j}^{\mathrm{KS}}(\mathbf{r})=\varepsilon_{j}^{\mathrm{KS}} \psi_{j}^{\mathrm{KS}}(\mathbf{r})
\end{array}
$$

where $\mathbf{r}$ and $\mathbf{r}^{\prime}$ are the coordinates of the $j$ and $j^{\prime}$ electrons, respectively, and $m_{e}$ is the mass of electron. $\hbar$ is the Plank constant and $q$ is the elementary charge. $\psi_{j}^{\mathrm{KS}}\left(\mathbf{r}_{j}\right)$ is the auxiliary non-interacting single-particle wavefunction. $\varepsilon_{j}^{\mathrm{KS}}$ is the orbital energy of the auxiliary wavefunction of electron $j$. The first term in the left side of equation is the kinetic energy operator and the second term is the Hatree energy operator. $V_{e n}$ is the ionic potential, and $V_{x c}(\mathbf{r})=\partial E_{x c}[\rho] / \partial \rho$ is the exchangecorrelation potential. $E_{x c}[\rho]$ is the exchange-correlation functional

$$
E_{x c}[\rho]=\frac{1}{2} \iint \rho(\mathbf{r}) \frac{\rho_{x c}\left(\mathbf{r}, \mathbf{r}^{\prime}\right)}{\left|\mathbf{r}-\mathbf{r}^{\prime}\right|} d \mathbf{r} d \mathbf{r}^{\prime}
$$

which is the Coulomb interaction between an electron at $\mathbf{r}$ and its exchange-correlation hole $\rho_{x c}\left(\mathbf{r}, \mathbf{r}^{\prime}\right)$ at $\mathbf{r}^{\prime}$. If we know exactly $\rho_{x c}\left(\mathbf{r}, \mathbf{r}^{\prime}\right), E_{x c}[\boldsymbol{\rho}]$, the total electron density and the total energy will be exact. Mapping the many-electron SE onto the single-particle SEs reduces the computation scale to $N^{3}$, which makes many studies possible including the crystal structure prediction.

The current DFT implemented in many codes is becoming more reliable and robust with the progress in condensed matter sciences ${ }^{23,24}$. New $2 \mathrm{D}$ crystals with various compositions and structural characteristics are continuously being predicted with the accurate structure search techniques based on DFT. Several crystal structure prediction methods are available. Searching manually among layered materials for new $2 \mathrm{D}$ materials is a practical way, by which several 2D materials were predicted ${ }^{25-29}$. However, it is usually confined to the known layered materials. To broaden the scope and to put it on 
a more physical basis, more advanced techniques for exploring the potential energy landscapes should be employed. The simulated annealing method probes the configurational space at each temperature during annealing using either a Monte Carlo or a molecular dynamics scheme, which allows to locate the global minimum structure given appropriate procedure. However, this method is confined from being applied to 2D systems since 2D systems always have ground-state bulk counterparts. The evolutionary algorithm method sets up the initial structures with random arrangements of atoms and let the structure evolve by mimicking the Darwinian evolution. Energetically favored candidate structures are likely to be chosen to create offspring structures by two operators, namely, the 'crossover' and 'mutation' operators. The first combines the current structural features of the parent structures and the latter introduces a Monte Carlo move with several random ionic displacements in the parent structures. Thus, structures at the energy minima can be predicted. However, the evolutionary algorithm method also suffers the inefficiency on 2D systems. But it can be extended specially to $2 \mathrm{D}$ systems by techniques like constraining the thickness of the structures ${ }^{30,31}$. The topological modelling methods have been very efficient to enumerate and predict new structures with very little amount of calculations under symmetry consideration ${ }^{32,33}$. This method can also be applied to the structure search of 2D systems. It is also noted that the organic 2D systems can be simply constructed using bottom-up strategies given the proper selection of units and linkers ${ }^{34-36}$, and the method gives more freedom to tune the structures and properties.

Besides, doping, defects, and alloying in semiconductors introduce complex variations to the microscopic structure that many unique properties depend on. Such detailed structural information, which relates to the order-disorder problem, some times, can only be understood with the help of simulations. The process to obtain the structures usually involves the evaluation of the energies of the enormous number of enumerated structures. However, this is very heavy and becomes unaffordable with the increasing size of supercell, though the system symmetry can be used to reduce the number of configurations. Another strategy is to parameterize the interactions using energies of small supercells and use the parametric interaction model to predict structures in any supercell. One of such widely used methods is the cluster expansion (CE) approach, which parametrizes the energy of structures as a polynomial in the occupation variables:

$$
E(\sigma)=\sum_{\alpha} m_{\alpha} J_{\alpha}\left\langle\prod_{i \in \alpha^{\prime}} \sigma_{i}\right\rangle
$$

where $\sigma_{i}$ is the occupation variables of site $i, \alpha$ is a set of cluster and $\alpha^{\prime}$ are that all symmetric equivalents to $\alpha$, and $J_{\alpha}$ is the expansion coefficient of the cluter $\alpha$. The advantage of $\mathrm{CE}$ approach is that it converges rapidly and the energy of any configuration can be immediately calculated once the cluster expansion has been constructed. The shortage of CE approach is that it fails for systems with abrupt changes of charge states.

\subsection{Prediction of photocatalytic properties}

As discussed in Section 1, photocatalytic applications require special electronic and optical properties. The most important properties that qualify a crystal for photocatalytic water splitting include the suitable bandgap, band edge levels, optical absorption, and charge carrier mobility. These properties can be accurately calculated by DFT simulations.

In the KS Hamiltonian (see Eq. 5), everything is known except for the exchange-correlation functional $E_{x c}$ (Eq. 6). Therefore, the accuracy of everything in DFT is naturally undertaken by $E_{x c}$. Kohn and Sham first proposed a simple local density approximation (LDA) to $E_{x c}$ :

$$
E_{x c}^{\mathrm{LDA}}[\rho(\mathbf{r})]=\int \varepsilon_{x c}^{\mathrm{HEG}}(\rho) \cdot \rho(\mathbf{r}) d \mathbf{r}
$$

where $\varepsilon_{x c}^{\mathrm{HEG}}(\rho)$ (known exactly) is the exchange-correlation energy per unit volume of homogeneous electron gas $(\text { HEG })^{37-39}$. LDA works well for metallic systems with slowly varying electron densities but not for semiconductors and insulators, for which bandgaps are substantially underestimated. The failure comes from the fact that DFT doesn't adopt virtual orbitals and LDA totally neglects the nonlocal effects. The later established adiabatic connection formula ${ }^{40-44}$ regarding to the exchange-correlation hole provides a rigorous way to improve the exchange-correlation functionals and thereafter a pool of functionals were developed. The generalized gradient approximation (GGA) ${ }^{45,46}$ takes into consideration of the inhomogeneity of electron density and is generally more accurate than LDA. Higher order derivatives of the electron density included in meta-GGA functionals can be helpful to the improvement of chemical accuracy on systems with sharp changes of electron density. However, all the GGA and meta-GGA functionals are just corrections to LDA and the same to LDA at zero density gradient. Thus, the exchangecorrelation holes of GGAs and meta-GGAs are inherently localized. The delocalized effects can only be accounted by replacing some of the exchange energy with the exact Foch exchange energy based on the adiabatic connection. The hybrid functionals mix the nonlocal Fock exchange with the local or semilocal DFT exchange, which can significantly improve the accuracy of electronic structures ${ }^{47,48}$. Similar to GGAs, there are also many versions of hybrid functionals available. It is worth to note that the chemistry society prefers to formulate semi-empirical parameterized functionals, while the physics society prefers to develop 'parameter-free' functionals fitted to the quantum Monte-Carlo results or physical laws. The former is highly accurate within its training set but with less trans- 
ferability, and the later may be not so accurate but is highly transferable.

There are other drawbacks due to that DFT does not adopt the virtual orbitals. For systems (materials with localized $d$ or $f$ electrons) with orbital-dependence strong correlation interactions, DFT fails to describe the localized and delocalized electrons equally well. The DFT $+\mathrm{U}$ methods can be employed to tackle this problem by adding a Hubbard-type Coulomb repulsion term to the KS Hamiltonian. Besides, the longdistance van der Waals interaction due to the instantaneous excitations is also neglected by DFT. Corrections can be made empirically ${ }^{49,50}$ or $a b$ initio $^{51,52}$. The correction to binding energy is about $0.1 \mathrm{eV} /$ atom $^{53,54}$, but the correction to the electronic structure is very limited.

DFT is for occupied orbitals and there is no rigorous meaning for the unoccupied orbitals. The above functionals and corrections do not address the problem of quasiparticle (QS) energies. For the accurate description of quasiparticle energies and excitation spectra, this usually needs to go beyond DFT. The quasiparticle energies can be determined by the GW method from

$$
\begin{array}{r}
\left\{-\frac{\hbar^{2}}{2 m_{e}} \nabla_{j}^{2}+\int \sum_{j^{\prime} \neq j} \psi_{j^{\prime}}^{\mathrm{QS}}\left(\mathbf{r}^{\prime}\right) \frac{q^{2}}{\left|\mathbf{r}-\mathbf{r}^{\prime}\right|} \psi_{j^{\prime}}^{\mathrm{QS}}\left(\mathbf{r}^{\prime}\right) d \mathbf{r}^{\prime}+\right. \\
\left.V_{e n}(\mathbf{r})\right\} \psi_{j}^{\mathrm{QS}}(\mathbf{r})+\int d^{3} \mathbf{r}^{\prime} \Sigma\left(\mathbf{r}, \mathbf{r}^{\prime} ; \varepsilon_{j}^{\mathrm{QS}}\right) \psi_{j}^{\mathrm{QS}}(\mathbf{r})=\varepsilon_{j}^{\mathrm{QS}} \psi_{j}^{\mathrm{QS}}(\mathbf{r})
\end{array}
$$

where the frequency-dependent self-energy operator $\Sigma$ is

$\Sigma\left(\mathbf{r}, \mathbf{r}^{\prime} ; \omega\right)=\frac{i}{4 \pi} \int_{-\infty}^{\infty} e^{i \omega^{\prime} \delta} G\left(\mathbf{r}, \mathbf{r}^{\prime}, \omega+\omega^{\prime}\right) W\left(\mathbf{r}, \mathbf{r}^{\prime}, \omega^{\prime}\right) d \omega^{\prime}$

where $\omega$ is the frequency, $\delta$ is the Dirac delta function. $G$ is the Green's function, and $W$ is the screened Coulomb interaction. The GW thus achieves the dynamical screening of electron-electron interactions, and the quasiparticle band structure can be obtained.

GGAs systematically underestimate the bandgaps of 2D semiconductors. The bandgap of single-layer $\mathrm{MoS}_{2}$ ranges from 1.58 to $1.78 \mathrm{eV}$ by different GGA functionals ${ }^{57}$, which are smaller than the experimental bandgap about $1.8 \mathrm{eV}^{61}$. The bandgap of Phosphorene is also underestimated by as much as $0.5 \mathrm{eV}^{62}$. The hybrid functionals including the nonlocal Fock exchange can give bandgaps much closer to the experimental data. The Heyd-Scuseria-Ernzerhof (HSE06) hybrid functional ${ }^{63,64}$ gave a bandgap of $1.49 \mathrm{eV}$ for Phosphorene, which is comparable with the experimental bandgap $1.45 \mathrm{eV}^{62}$. The GW methods, which are expected to give more accurate bandgaps, while substantially overestimate the bandgaps of 2D semiconductors. The bandgaps of single-layer $\mathrm{MoS}_{2}$ and Phosphorene from the GW methods are 2.75-2.8 $\mathrm{eV}^{58,65,66}$ and 2.0-2.3 $\mathrm{eV}^{62}$, respectively. It is argued that the $\mathrm{GW}$ bandgap is the quasiparticle fundamental bandgap, while

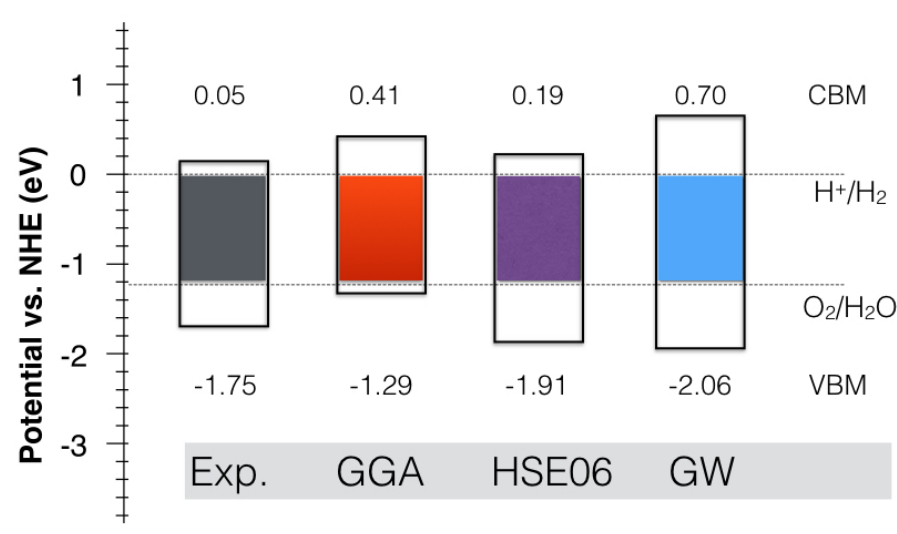

(a)

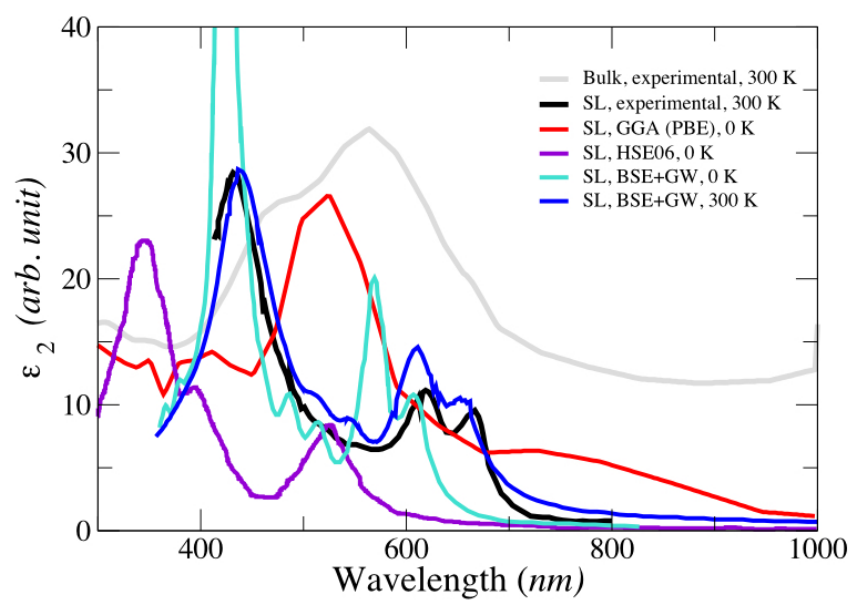

(b)

Fig. 3 The experimental and calculated band edge positions of the single-layer $\mathrm{MoS}_{2}$ are shown in (a). The experimental data is from Refs 55,56. The levels calculated by GGA and HSE06 are from Ref. 57. The GW results are from Ref. 58. The imaginary parts of the frequency-dependent dielectric functions of $\mathrm{MoS}_{2}$ are plotted in (b), for which the experimental data of bulk is from Ref. 59, the data of GGA and HSE06 is from Ref. 57, and the experimental and $\mathrm{BSE}+\mathrm{GW}$ data for the single layer is from Ref. 60.

the experimental observed is usually the optical bandgap. The 2D materials have reduced Coulomb screening and hence high exciton binding energies. Thus, the optical bandgap is significantly smaller than the fundamental bandgap. Considering the exciton effects, the optical bandgaps given by the Bethe-Salpeter equation (BSE) ${ }^{67}$ based on the GW quasiparticle band structures are consistent with the experimental bandgaps ${ }^{68,69}$. However, the large fundamental bandgaps haven't been confirmed by experiments yet.

Appropriate band edge levels are very important for driving 
the redox reactions. The accuracy of the exchange-correlation functionals as regard to the band energy levels is similar to their performances on the bandgap prediction. As can be seen in Fig. 3(a), the CBM and VBM levels of single-layer $\mathrm{MoS}_{2}$ calculated by GGA are higher than those of their experimental counterparts by 0.36 and $0.46 \mathrm{eV}$, respectively. These discrepancies are reduced to 0.14 and $0.16 \mathrm{eV}$, respectively, by the HSE06 hybrid functional. However, CBM and VBM levels predicted by the GW method deviate from the experimental levels more than those of the GGA functional, which is possibly due to the incomplete selfconsistency of the GW calculation and the neglect of exciton effects.

The predicted optical absorption spectra of single-layer $\mathrm{MoS}_{2}$ are plotted in Fig. 3(b) in comparison with experimental spectra. As can be seen, the shape of the imaginary part of the dielectric function (corresponding to the experimental optical absorption spectrum) calculated by HSE06 show similar characteristics with the experimental spectrum but is shifted to the short-wavelength side. This agrees well with the fact that HSE06 overestimates the bandgap of single-layer $\mathrm{MoS}_{2}$. The $\mathrm{BSE}+\mathrm{GW}$ method taking consideration of the temperature effect gives the best match with the experimental data. While, the spectrum from GGA bears more similarities to that of bulk $\mathrm{MoS}_{2}$ rather than the single layer, which suggests that the nonlocal effects are very important for the accurate description of optical properties in the single layer.

Overall, current state-of-the-art simulations can provide accurate descriptions for the structural, electronic, and optical properties of 2D materials. Computational screening of the 2D materials for photocatalysis is practical, fast, economical, and powerful. In the following Section, we summarize the recent progress on 2D photocatalysis. Meanwhile. we address on the methods to tune the electronic and optical properties of 2D materials and how DFT simulations can be used to facilitate this process.

\section{Discussion}

\subsection{Electronic structure}

2D materials can always find their bulk counterparts, and can be obtained from direct exfoliation of the bulk materials. These kinds of materials have layered structures with weak interactions in between. The interaction energy (or formation energy of single layer) between the layers is an important parameter to measure the isolability of the bulk material. Such weak interactions due to correlated electron interactions can be van der Waals forces or hydrogen bonding (in materials like layered metal hydroxides ${ }^{33,70}$ ). DFT based simulations can be used to examine the stability of possible 2D materials by calculating their free energies and lattice dynamics.

Table 1 lists the formation energies of some 2D materials.
Some of them have been fabricated and some are just predicted. It can be seen that the formation energies are typically less than $1 \mathrm{eV} /$ atom, which suggests the weak binding between the layers. The production of single layers needs to get ride of or prevent the interlayer binding by physical or chemical methods. For example, metal chalcogenides can be separated into single layers by intercalation in a solvent. By selecting an appropriate solvent that has an interaction energy with the layers greater than that between the layers in the bulk, the separation process can become much easier ${ }^{71}$.

Table 1 Formation energy $\mathrm{E}_{f}$ of single-layer materials from bulk materials.

\begin{tabular}{|c|c|c|}
\hline Material & $\overline{\mathrm{E}_{f}(\mathrm{eV} / \text { atom })}$ & Refs \\
\hline AlN & $0.51 \sim 0.58$ & 25 \\
\hline $\mathrm{GaN}$ & $0.42 \sim 0.76$ & 25 \\
\hline AlP & 0.31 & 25 \\
\hline AlAs & 0.22 & 25 \\
\hline $\mathrm{GaN}$ & $0.44 \sim 0.45$ & 25 \\
\hline GaAs & 0.32 & 25 \\
\hline $\mathrm{MoS}_{2}$ & 0.076 & 72 \\
\hline $\operatorname{InX}(x=S, S e, T e)$ & $0.068 \sim 0.098$ & 72 \\
\hline $\mathrm{GaX}(\mathrm{X}=\mathrm{s}, \mathrm{Se}, \mathrm{Te})$ & $0.058 \sim 0.068$ & 72 \\
\hline$\alpha-\mathrm{ZrNX}(\mathrm{X}=\mathrm{Cl}, \mathrm{Br}, \mathrm{I})$ & $0.042 \sim 0.044$ & 72 \\
\hline$\alpha$-HfNX $(\mathrm{X}=\mathrm{Cl}, \mathrm{Br}, \mathrm{I})$ & $0.042 \sim 0.046$ & 72 \\
\hline$\alpha$-TiNX $(\mathrm{X}=\mathrm{Cl}, \mathrm{Br}, \mathrm{I})$ & $0.039 \sim 0.044$ & 72 \\
\hline Graphene & 0.063 & 73 \\
\hline Silicene & 0.76 & 74 \\
\hline Germanene & 0.99 & 74 \\
\hline Arsenene & 0.0896 & 26 \\
\hline Antimonene & 0.086 & 26 \\
\hline $\mathrm{TM}_{2} \mathrm{C}(\mathrm{TM}=\mathrm{Mo}, \mathrm{Tc}, \mathrm{OS})$ & $0.61 \sim 0.94$ & 29 \\
\hline
\end{tabular}

We collected the known inorganic 2D materials in the periodic table shown in Fig. 4. The involved elements in the inorganic 2D systems are the $p$-block and $d$-block elements. We tentatively classify them into the $2 \mathrm{D}$ elements, $2 \mathrm{D}$ carbides/nitrides, and 2D chalcogenides. The elemental 2D systems are usually stabilized by the $p \pi$-bonding network, which normally induces small bandgaps or zero bandgaps. The 2D chalcogenides are stabilized by the $p-d$ hybridization, and usually have enough bandgaps for the photocatalytic reaction. As depicted in Fig. 5, the consequence of this hybridization leads to the valence band maximum (VBM) and conduction band maximum (CBM) to be controlled by the $d$ states of the transition metals. The outermost $d$ orbitals are incompletely filled with electrons, so they can easily give and take electrons. This makes the 2D chalcogenides ideal candidates for catalysis. As shown in Fig. 5, the bandgap of single-layer transitional metal dichalcogenides (TMDCs) is determined by the energy levels of two antibonding crystal orbitals, which in turn can be tuned by the $d$ orbital splitting energy. The degeneracy of $d$ orbitals loses in a non-spherical Coulomb potential, and the bigger the 
difference of the electronegativity between the transition metal atoms and the anions, the higher the $d$ orbital splitting. This is manifested in the electronic structures of the TMDCs ${ }^{75,76}$.

Fig. 6 shows the calculated band alignment for a typical series of inorganic layered materials, $\mathrm{MX}_{2}(\mathrm{M}=\mathrm{W}, \mathrm{Mo}$, and $\mathrm{X}=\mathrm{S}, \mathrm{Se}, \mathrm{Te}$ ), as a function of the number of layers. The plot shows a lot of interesting trends. The band alignments of single-layer $\mathrm{MX}_{2}$ show remarkable differences from the bulk materials. The CBM comes from the antibonding states of Mo $-d_{z^{2}}, \mathrm{P}-p_{x}$ and P- $p_{y}$ orbitals, while the VBM is dominated by the bonding states of Mo- $d_{x^{2}-y^{2}}$ and Mo- $d_{x y}$ orbitals. With decreasing number of layers, the quantum confinement effects (QCE) make the population of the antibonding states of Mo$d_{z^{2}}, \mathrm{P}-p_{x}$ and $\mathrm{P}-p_{y}$ orbitals more difficult, while make the population of their bonding states easier. So, the level of CBM mainly from the Mo- $d_{z^{2}}$ state will be pushed up due to QCE, and the level of VBM will be pushed down. In comparison, Fig. 7 shows the band alignment for a typical organic layered material, the covalent triazine framework $(\mathrm{CTF})^{34}$, which is a series of monolayer and multilayer covalent triazine frameworks. It can be seen that the energy levels of the bands are not strongly affected by the change of number of layers. For AA stacking, the energy levels almost do not change with the increasing number of layers. This is because that the organic units with closed shells are more chemically inert. Generally speaking, organic layered materials show weaker layer interactions than the inorganic layered materials.

2D materials can be metallic or semiconducting, but insulating 2D materials have not been found. The electronic structures of elemental 2D materials are governed by their $p$ states. They are metals when $p$ orbitals are incompletely occupied, such as Borophene, Graphene, Silicene, and Germanene, but they are semiconductors when $p$ orbitals are fully populated like Phosphorene. Surface functionalization can be employed to tune their electronic structures as the dangling bonds are in favor of binding molecules or adatoms ${ }^{54}$. As regard to the binary and ternary 2D materials, the bandgap not only depends on the elemental orbitals, but also depends on other things like the coordination, and the stacking of layers as mentioned above.

Some peculiar properties have also been found and investigated in $2 \mathrm{D}$ materials after the successful isolation of Graphene $^{11}$. These phenomena are size-dependent ${ }^{78}$ and sensitive to external perturbations. Examples can be the bandgap closure and opening ${ }^{61}$, semiconductor-metal transition, giant spin-orbit splitting ${ }^{79,80}$, spin-valley coupling ${ }^{81-83}$ and outof-plane Zeeman effects ${ }^{84}$, and DFT has shown its power in studying these properties. Concerning photocatalytic properties, the changes of charge carrier concentrations, conductivity, bandgap and Fermi level are critical for possible photocatalytic water splitting, and these can also be successfully described by DFT simulations. Many 2D materials with proper electronic structures have been tested for photocatalytic applications. Table 2 summarizes the photocatalytic properties of some 2D materials. 2D photocatalysis show improved catalyzing properties compared to their bulk counterparts, which is due to the unique electronic structures and abundant active sites. Fig. 8 illustrates the bulk form, single-layer $\mathrm{MoS}_{2}$, and the single-layer $\mathrm{MoS}_{2}$ cocatalyzed by nanoparticles. The bulk with compact layers (Fig. 8(a)) is almost inert in catalyzing water splitting due to the lack of active sites. The single layer (Fig. 8(b)) exposes abundant active edge sites and surface sites. Thus, the single-layer $\mathrm{MoS}_{2}$ produces higher $\mathrm{H}_{2}$ yields (see Table 2), which is far better than the bulk. Besides, the single-layer $\mathrm{MoS}_{2}$ and CdS cocatalyst system (illustrated in Fig. 8(c)) exhibits significantly enhanced catalytic activity. As shown in Table 2, the $\mathrm{H}_{2}$ yield of the cocatalyst (1472 $\mu \mathrm{mol} / \mathrm{h} / \mathrm{g})$ is almost 30 times of that of the pure single-layer system.

As discussed above, the electronic structures of 2D materials can be variant under different conditions. The techniques that can be used to tune their electronic and photocatalytic properties naturally come from these conditions. These techniques that will be introduced below can effectively engineer 2D materials for photocatalytic applications by introducing physical or chemical perturbations to the pristine systems. Such perturbations can also be virtually performed by DFT simulations on 2D materials to effectively explore their photocatalytic property spaces.

\subsection{Bandgap engineering (Straining, doping and defect engineering)}

The efficiency of a photocatalyst strongly depends on its ability to harvest the light energy. Exciting an electron from VB to CB can only occur with suitable bandgap under specific light sources. Suitable bandgaps for visible light harvesting should be around $2.0 \sim 2.2 \mathrm{eV}^{102}$. Bandgap engineering refers to the manipulation of bandgap by varying the chemical and/or physical conditions. It is a powerful technique that is widely used in the semiconductor industry.

Bandgap engineering via mechanical strain is an effective and practical way to tune the physical and chemical properties of semiconductors, which has been exemplified by many studies $57,103-105$. The applied strain can directly change the overlap of orbitals and induce spatial charge redistribution. However, there is no general rule that applies for the effects of strain on electronic structures of materials as the consequences of the applied strain vary with the specific bonding and local symmetry of ions.

Single-layer materials can sustain high mechanical strain. Graphene can hold up to an uniaxial strain of $25 \%,{ }^{106}$ and Phosphorene can stand with $30 \%$ strain ${ }^{107}$. This grants us a great freedom for bandgap engineering via the applied me- 



Fig. 4 Periodic table summarizing the 2D materials that have been found experimentally or theoretically.
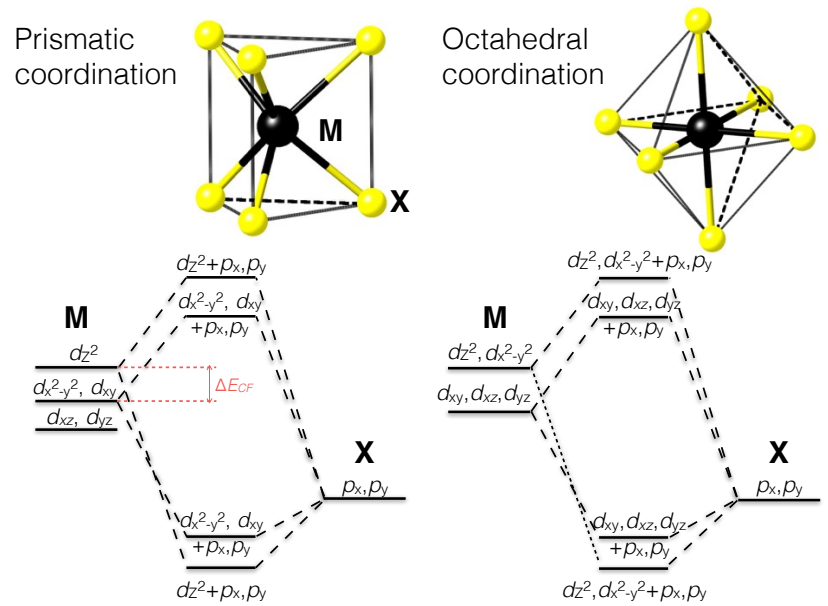

Fig. 5 Schematic illustration of the states of $\mathrm{MX}_{2}$ single layers bonded with $p$ and $d$ orbitals. Different coordination environment causes the different splitting of $d$ states, which determines the bandgaps and energy levels of VB and CB.

chanical strain. The transition from semiconductor to metal was even achieved in the single-layer $\mathrm{MoS}_{2}$ and Phosphorene ${ }^{105}$. The Raman scattering shifts ${ }^{108}$, direct-to-indirect and indirect-to-direct bandgap transition ${ }^{26,57,76,109}$ and conducting pattern change ${ }^{104}$, etc. were also found to be viable by applying strain. Especially, the applied mechanical strain has influential consequences on the photocatalytic properties of semi-

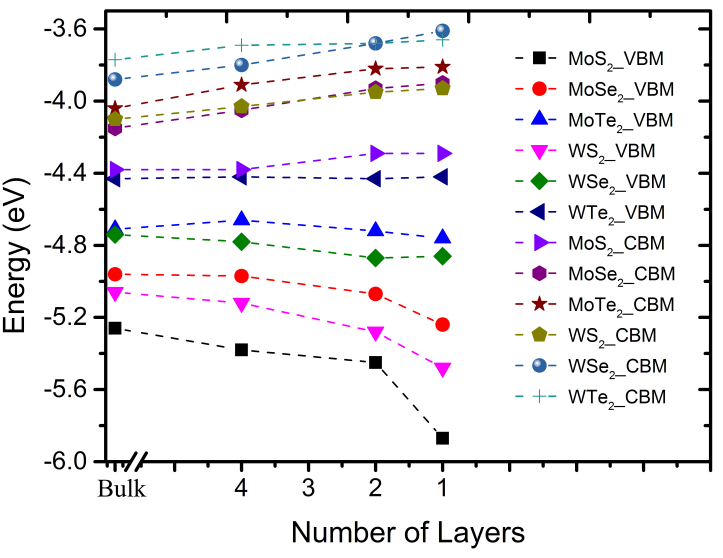

Fig. 6 Band edge positions of $\mathrm{MX}_{2}$ ( $\mathrm{M}$ for $\mathrm{Mo}$ and $\mathrm{W}, \mathrm{X}$ for $\mathrm{S}, \mathrm{Se}$ and $\mathrm{Te}$ ) referenced to the vacuum level as a function of the number of layers calculated by PBE functional. Reproduced from Ref. 77

conductors. For instance, Phosphorene can be engineered as a good candidate for overall water splitting with appropriate bandgaps and band edge alignments at certain $\mathrm{pH}$ value. Fig. 9 shows the bandgap and the band edges of Phosphorene as a function of the applied strain.

It is not a difficult task to apply mechanical strain either in experiments or in practical applications. One can achieve strain engineering by choosing different substrates, bending of 
Table 2 Specific surface area (S.A.), conduction band (CB) level, valence band (VB) level, bandgap (E E $_{g}$ and photocatalytic properties of 2D materials.

\begin{tabular}{|c|c|c|c|c|c|c|c|c|}
\hline Materials & $\begin{array}{l}\text { S.A. } \\
\left(\mathrm{m}^{2} / \mathrm{g}\right)\end{array}$ & $\begin{array}{l}\mathrm{CB}(\mathrm{eV}) \\
(\mathrm{eV})\end{array}$ & $\begin{array}{l}\mathrm{VB} \\
(\mathrm{eV})\end{array}$ & $\begin{array}{l}\mathrm{E}_{g} \\
(\mathrm{eV})\end{array}$ & Light Source & cocatalyst & $\begin{array}{l}\mathrm{H}_{2} \text { yield } \\
(\mu \mathrm{mol} / \mathrm{h} / \mathrm{g})\end{array}$ & Refs \\
\hline$g-\mathrm{C}_{3} \mathrm{~N}_{4}$ & 10 & -3.37 & -6.07 & 2.7 & $\lambda>420 \mathrm{~nm}$ & - & 3.2 & 85 \\
\hline$g-\mathrm{C}_{3} \mathrm{~N}_{4}$ & - & -3.37 & -6.07 & 2.7 & $\lambda>300 \mathrm{~nm}$ & $\mathrm{Pt}$ & 2368 & 85 \\
\hline Graphene & - & -4.42 & -4.42 & 0 & $300 \mathrm{~W} \mathrm{Xe}$ & $\mathrm{CdS}$ & 1050 & 86 \\
\hline $1 \mathrm{H}-\mathrm{MoS}_{2}$ & - & -4.03 & -5.74 & 1.75 & - & - & - & 57 \\
\hline $1 \mathrm{~T}-\mathrm{MoS}_{2}$ & - & - & - & 0 & $100 \mathrm{~W}$ halogen & - & 26000 & 87 \\
\hline $1 \mathrm{~T}-\mathrm{MoSe}_{2}$ & - & - & - & 0 & $100 \mathrm{~W}$ halogen & - & 62000 & 88 \\
\hline $1 \mathrm{~T}-\mathrm{WS}_{2}$ & - & -4.4 & -4.4 & 0 & $300 \mathrm{~W} \mathrm{Xe}$ & $\mathrm{TiO}_{2}$ & 2570 & 89 \\
\hline $2 \mathrm{H}-\mathrm{WS}_{2}$ & - & -3.6 & -5.6 & $\sim 2.0$ & $300 \mathrm{~W} \mathrm{Xe}$ & $\mathrm{TiO}_{2}$ & 225 & 89 \\
\hline $\mathrm{MoS}_{2}$ & - & - & - & - & $\lambda>420 \mathrm{~nm}$ & $\mathrm{CdS}$ & 1472 & 90 \\
\hline $\mathrm{SnS}_{2}$ & - & - & - & $2.08 \sim 2.55$ & $300 \mathrm{~W}$ Xe & - & 1060 & 93,94 \\
\hline $\mathrm{GaX}(\mathrm{X}=\mathrm{S}, \mathrm{Se}, \mathrm{Te})$ & & $-3.58 \sim-3.38$ & $-6.77 \sim-5.75$ & $2.22 \sim 3.19$ & - & - & - & 72 \\
\hline $\operatorname{InX}(\mathrm{X}=\mathrm{S}, \mathrm{Se}, \mathrm{Te})$ & & $-4.02 \sim-3.44$ & $-7.18 \sim-6.13$ & $2.20 \sim 2.71$ & - & - & - & 72 \\
\hline CTF-0 & - & -3.27 & -6.59 & 3.32 & - & - & - & 34 \\
\hline$\left(\mathrm{C}_{4} \mathrm{H}_{9} \mathrm{NH}_{3}\right)_{2} \mathrm{PbBr}_{4}$ & - & - & - & 3.01 & - & - & - & 95 \\
\hline Phosphorene & - & -4.15 & -5.94 & 1.79 & - & $\mathrm{pH}=8.0$ & - & 62 \\
\hline$\alpha-(\mathrm{P}, \mathrm{As}, \mathrm{Sb}, \mathrm{Bi})$ & - & -1.82 & -4.66 & $0.99 \sim 2.62$ & - & - & - & 96 \\
\hline$\beta-(\mathrm{P}, \mathrm{As}, \mathrm{Sb}, \mathrm{Bi})$ & - & -1.22 & -3.82 & $0.36 \sim 1.83$ & - & - & - & 96 \\
\hline $\mathrm{CuWO}_{4}$ & - & $\sim-4.0$ & $\sim-5.2$ & $\sim 1.2$ & - & - & - & 97 \\
\hline $\mathrm{APX}_{3}$ & - & $-4.03 \sim-3.07$ & $-7.01 \sim-5.80$ & $1.77 \sim 3.94$ & - & - & - & 98 \\
\hline $\mathrm{ZnIn}_{2} \mathrm{~S}_{4}$ & 103 & - & - & 2.3 & $300 \mathrm{~W}$ Xe & - & 57 & 99 \\
\hline
\end{tabular}

a flexible substrate ${ }^{103}$, direct mechanical contact or by using local thermal expansion ${ }^{110}$. Actually, the out-of-plane strain is also possible and can be introduced during the preparation and characterization processes, as can be seen in the previous literature $^{111,112}$. The idea is to suspend the single layer on prepatterned substrate containing an array of circular holes. The $\mathrm{z}$-axis strain can be introduced by pushing the singlelayer on the hole or compression in sandwich configuration. The effects of the out-of-plane strain is predicted in reverse to the effects of in-plane stains ${ }^{57}$, which is confirmed by experiment $^{113}$.

Doping refers to the introduction of foreign elements into the lattice of the host materials. Doping with electron donors (known as $\mathrm{N}$-type doping) can increase the number of negative charge carriers within the semiconductors. While doping with an electron acceptor (known as a P-type dopant) will create a deficiency of electrons. Doping is an alternative and more powerful technique to prepare visible light-driven photocatalysis. The introduction of a trace amount of impurities into semiconductors can effectively increase the number of charge carriers. Dopants can form impurity levels in the bandgap of the host materials, resulting in positive visible light response. However, doping may bring up with recombination centers between photogenerated electrons and holes, which will significantly affect the photocatalytic activity of the host material. Anyway, doping with suitable dopants have been proved to be effective in improving the activities of visible light-driven photocatalysis. For instance, it is found that doping $\mathrm{Cr}$ and $\mathrm{Fe}$ in $\mathrm{La}_{2} \mathrm{Ti}_{2} \mathrm{O}_{7}$ can induce enhanced $\mathrm{H}_{2}$ evolution under visible light ${ }^{114}$. The cation-anionic co-doping in $\mathrm{SrZrO}_{3}$ is found to reduce the bandgap and its electronic band position becomes more appropriate for the visible-light photocatalysis ${ }^{115}$. 


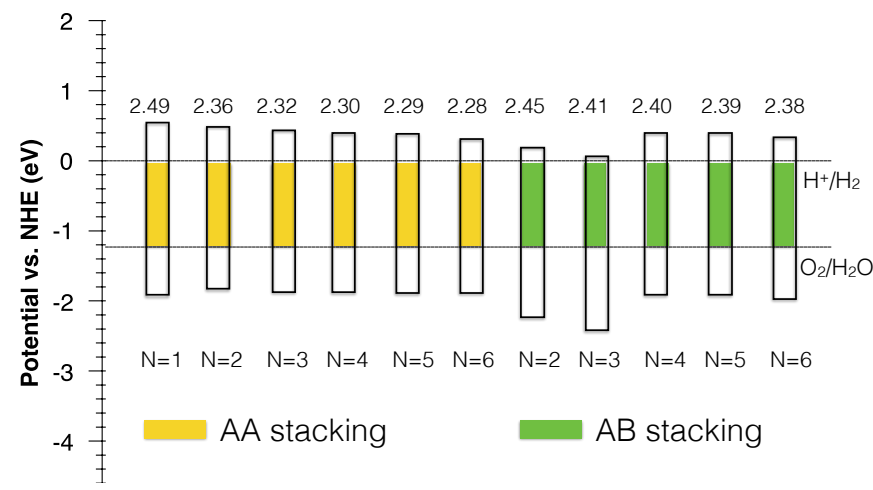

Fig. 7 Band edge positions of CTF-0 with AA stacking and AB stacking. The potential is referenced to the normal hydrogen electrode (NHE) in electron volts. The redox potentials of water splitting are shown for comparison. Reproduced from Ref. 34.

In a recent work by Jia et.al., ${ }^{86} \mathrm{~N}$-doped Graphene (NGraphene) co-catalyzed by $\mathrm{CdS}$ was synthesized by calcination. The photocatalytic properties of the synthesized $\mathrm{N}-\mathrm{Graphene} / \mathrm{CdS}$ for hydrogen production from water was shown to be dramatically improved under visible-light irradiation. The results show that the catalyst with N-Graphene exhibits much higher photocatalytic activity than the pure CdS. It is also found that the photocurrent of the N-Graphene/CdS catalyst is also much higher than that of the sole $\mathrm{CdS}$, measured with a photoelectrochemical test device. The reason laying behind is that the radiative recombination of e-h pairs is reduced for the $\mathrm{N}-\mathrm{Graphene/CdS}$ photocatalyst. The excited electrons in the CdS prefer to move to the N-Graphene, and therefore, choosing the appropriate amount of N-Graphene is the key for maximizing the photocatalytic activity of the $\mathrm{N}$ Graphene/CdS photocatalyst. The photocatalytic activity of $\mathrm{N}-\mathrm{Graphene/CdS}$ is much higher than the GO/CdS composites, echoing the importance of choosing appropriate foreign elements. Besides, the N-Graphene/CdS photocatalyst also show higher robustness and the N-Graphene can act as a protective layer to prevent CdS from photocorrosion. Their findings show the inspiring results from doping 2D photocatalysis. Besides, alloying 2D semiconductor is another technique similar to doping. Single layers like $\mathrm{MoX}_{2}(\mathrm{X}=\mathrm{S}, \mathrm{Se}, \mathrm{Te})$ and $\mathrm{WX}_{2}(\mathrm{X}=\mathrm{S}, \mathrm{Se}, \mathrm{Te})$ have small lattice mismatch with each other. This provides opportunities for searching new phases with enhanced properties.

Besides, defects always exist in materials and may have remarkable effects on the photocatalytic properties. If we consider the change of Gibbs free energy $\Delta G$ of a system with $\mathrm{N}$ atoms, in association with the creation of the number of $N_{D}$ point defects

$$
\Delta G=N_{D} \cdot g_{D}-T \cdot \mathrm{k}_{B} \cdot \ln \left[\left(\mathrm{N}+N_{D}\right) ! /\left(N_{D} ! \mathrm{N} !\right] .\right.
$$

where $g_{D}$ is the defect formation energy per atom, then, in equilibrium, $N_{D}$ is always positive when $T>0 \mathrm{~K}$. Therefore, point defects inevitably exist in materials. The number of point defects increases with the increasing temperature in the Arrhenius' way. When point defects are populated, larger and extended defects can occur in favor of lowering the free energy. 2D materials should be more defective than their bulk counterparts since $g_{D}$ is lower in 2D materials. The reason is that there are more exposed surface atoms in $2 \mathrm{D}$ materials, which can migrate from the original lattice sites in a much easier way than in the bulk to form defects upon perturbations. Very recently, Zou et. al. ${ }^{116}$ studied the dislocations in 2D TMDCs. It is found that dislocations can be highly mobile in 2D TMDCs and the lattice can be ramarkably changed. The discovered generalized Stone-Wales (SW) rotation migration leads to significant displacements of several atoms. The occurrences of structural defects bring up with more chemical reactive sites. This is because that defects and disorder changes the equilibrium coordination numbers, which is expected to be effective to tune the activity of the reactive sites and affect the catalytic properties ${ }^{117}$. Besides, defects tend to lower the bandgap due to the introduction of new states in the forbidden band, which enhances the absorption of light. There have been some successful studies employing defect engineering. Especially, Ataca and Ciraci's study ${ }^{118}$ shows that the vacancy in the single-layer $\mathrm{MoS}_{2}$ helps capture free water molecules. The existence of vacancy favors the dissociation of water molecule, and the $\mathrm{O}$ atom keeps to be bonded to the vacancy, while the $\mathrm{H}$ atoms from water dissociation can diffuse readily and form $\mathrm{H}_{2}$ molecules spontaneously. In another study, Tay et al. ${ }^{119}$ induced the two-coordinated nitrogen vacancy in $g-\mathrm{C}_{3} \mathrm{~N}_{4}$ and detected a narrowed bandgap, which leads to an enhancement in solar absorption and hydrogen yields.

\subsection{Electrical and magnetic fields}

The electrons and holes will conduct in opposite directions in semiconductors on occasion there is an external electric or magnetic field. However, since the mass is not the same for electrons and holes, both carriers do not necessarily move with the same velocity under external fields. This creates the chances to reduce the recombination rates of photogenerated e-h pairs. For 2D TMDCs, it is found that external fields may induce material-dependent results, and the influences strongly vary with the number of layers ${ }^{120}$.

A perpendicular electric field on 2D TMDCs can reduce the bandgap, and bring about the closure of bandgap for some 
(a)

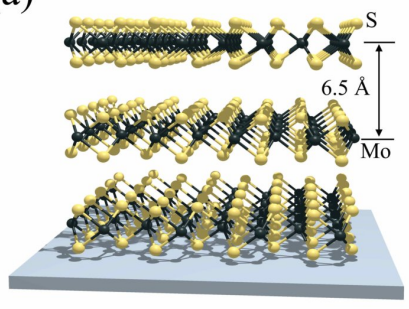

(b)

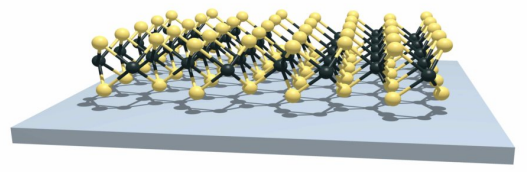

(c)

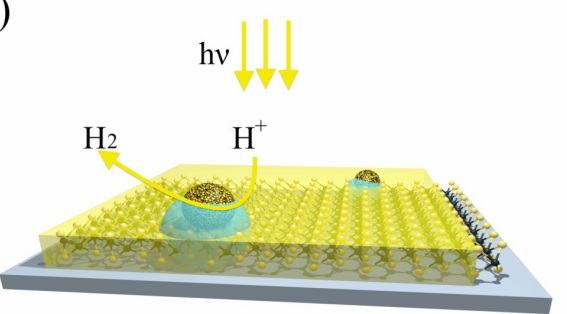

Fig. 8 Illustration of the (a) $2 \mathrm{H}-\mathrm{MoS}_{2}$ structure, (b) $1 \mathrm{H}-\mathrm{MoS}_{2}$ structure and (c) $1 \mathrm{H}-\mathrm{MoS}_{2}$ cocatalyzed by nano particles.
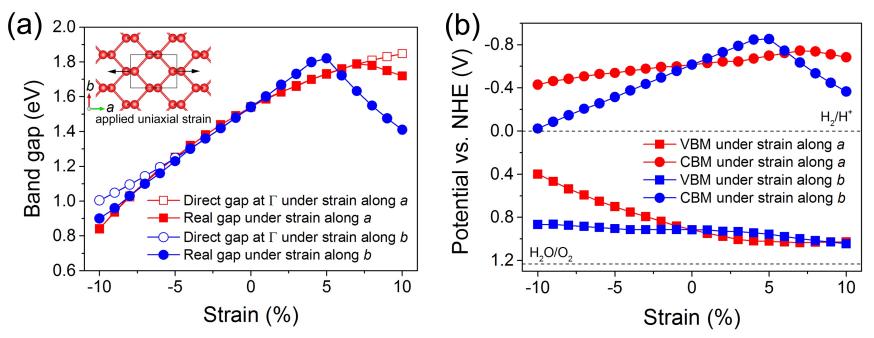

Fig. 9 The evolution of bandgap (a) and the band edges (b) of Phosphorene as a function of the applied strain using HSE06 functional. The inset in (a) shows the planar structure of Phosphorene and how the strain is applied. Reproduced from Ref. 62 .

structures ${ }^{110}$. Anyhow, the external fields require an extra input of power that is costly. A more efficient way is to create internal electrical and magnetic fields. These internal fields can be created by the solution/photocatalyst interfaces ${ }^{4,121}$, p-n junctions ${ }^{122,123}$, cocatalysis interfaces ${ }^{124,125}$, and polarization inside crystals.

There have been many successful examples in bulk photocatalysis. Recently, Jiang et al. ${ }^{126}$ found an internal polar electric field in $\mathrm{Bi}_{4} \mathrm{~V}_{2} \mathrm{O}_{11}$ using DFT calculations, which helps separate the photo-generated e-h pairs and plays an important role in the excellent photocatalytic activity of $\mathrm{Bi}_{4} \mathrm{~V}_{2} \mathrm{O}_{11}$.

Such cases in the bulk materials can be simply extended to 2D systems. Actually, in some cases, the working mechanism is similar to the cocatalyst approach that will be introduced below.

\subsection{Z-scheme and cocatalyst}

The idea of Z-scheme water splitting by linking two photosystems as shown schematically in Fig.2(b) was first proposed by Bard in $1979^{2}$. The two different photosystems are connected by the shuttle redox mediator. The visible light can be utilized more efficiently than in the one-step water splitting process since the energy barrier to drive each photocatalyst is reduced.

Since Bard ${ }^{2}$ proposed the concept and showed Z-scheme systems have significant advantages over conventional onestep systems, many studies have been conducted to combine appropriate materials into such a Z-scheme system. However, some inappropriate combinations may lead to the termination of water splitting. In those cases, the backward reactions involving redox reagents are thermodynamically favored, which proceed preferentially over the photocatalytic water splitting and suppress the gas evolution. Besides, the balance of the reduction reaction and oxidization reaction is much more difficult to achieve in $\mathrm{Z}$-scheme systems, since the two reactions have different favorable redox concentrations. However, there have been successes showing a stoichiometric $\mathrm{H}_{2}$ and $\mathrm{O}_{2}$ evolution can be well achieved via $\mathrm{Z}$-scheme photocatalysis.

Another similar scheme is the cocatalyst system, in which the light harvesting semiconductors are loaded with appropriate cocatalysis. This usually promotes the process of photocatalytic water splitting. Cocatalysis can provide more reactive sites and further catalyze the water splitting reactions. Appropriate cocatalysis can promote the charge separation and transport process and induce surface plasmonic resonance, which is the result of junctions/interfaces effects as illustrated in Fig. 8(c). The performances of pure 2D materials can be greatly improved with cocatalysis, which can be seen in Table 2. For instance, the $\mathrm{H}_{2}$ production rate of $g-\mathrm{C}_{3} \mathrm{~N}_{4}$ was enhanced by about 35 times by adding a small amount of $\mathrm{CdS}$ nano particles. The Fermi level of $\mathrm{CdS}$ is much higher than that of $g-\mathrm{C}_{3} \mathrm{~N}_{4}{ }^{85,127}$. This difference leads to an energy level offset, which subsequently causes band bending and the formation of space-charge region at the interface. Such phenomena have also been observed in other systems like the $\mathrm{Au} / \mathrm{MoS}_{2}{ }^{128}$ cocatalyst. When $2 \mathrm{D}$ semiconductors are integrated with cocatalysis to form nanocomposites, the band bending and space-charge region can facilitate the efficient charge separation. The induced surface plasmonic resonance will also facilitate the excitations and amplify the numbers of additional e-h pairs.

In the $\mathrm{Au} / \mathrm{MoS}_{2}{ }^{128}$ system, the space-charge region is 
charge free but the side on $\mathrm{MoS}_{2}$ is occupied by negative charges and the Au side is occupied by positive charges. A diploe occurs due to the charges distributed in a parallel manner. It is believed that the formation of space-charge region can facilitate e-h separation and reduce charge recombination. ${ }^{129}$ Under thermal equilibrium condition, further movement of charge carries is not favored by the dipole. However, when the thermal equilibrium is perturbed by the incident photons, the electric field in space-charge region will strictly promote the photoexcited electrons to pass the potential barrier while holes are left at $\mathrm{MoS}_{2}$ side. Besides, the plasmonic resonance of $\mathrm{Au}$ particles also leads to the enhanced production rate. the plasmonic resonance occurs when the free electrons on the surface of $\mathrm{Au}$ particles oscillate in phase with the varying electromagnetic field of the incident light. Subsequently, an electric field in the form of plasmonic energy will be generated by the free electrons, which has the same frequency as that of incident light. The induced plasmonic energy provides additional energy input for e-h excitation and separation within the nanocomposites. ${ }^{130,131}$ Meanwhile, the plasmonic heating effect also contributes to the improved photoactivity. ${ }^{132}$ It is worth noting that metallic $2 \mathrm{D}$ materials are more efficient in cocatalyzing than semiconductors. Semiconducting is not as reactive as metallic, and to have chemical interaction with cocatalysis, electrons must be promoted from bonding states to antibonding states.

DFT based simulations can facilitate the developments of the Z-scheme and cocatalyst systems by predicting the electronic structures. Using appropriate methods, accurate energy levels that are needed for designing the Z-scheme and cocatalyst systems can be obtained. Using DFT, Zhao et al. ${ }^{133}$ have successfully located the electron mediators and conductors in Z-scheme photocatalysis containing Graphene-based carbon nanostructures. Shiga et al. ${ }^{134}$ calculated the electronic structure of $\mathrm{SnS}$ and found that it can be used to construct a Z-scheme system for efficient overall water splitting. When tested experimentally, $\mathrm{SnS}$ was coupled with a photoanode and an efficient hydrogen production was obtained.

\subsection{Superlattice heterogeneous structures}

As discussed in Section 3.1, the electronic structure of 2D materials depends on the coordinations and stacking sequences, and therefore, construction of superlattices and heterostructures is another effective approach for improving the photocatalytic activity. The heterogeneous structures tend to have smaller bandgaps and help separate the e-h pairs. The design of appropriate superlattices and heterostructures can be efficiently facilitated by DFT based simulations by checking the stability, electronic structure, and optical properties.

Very recently, up to $20 \%$ maximum power conversion efficiency of Phosphorene and TMDCs heterostructures by strain engineering have been predicted ${ }^{135}$. The interfaces formed in superlattices and heterostructures can improve the photoinduced charge separation and provide more active sites. Many heterogeneous 2D materials have been synthesized with improved photocatalytic properties. Liao et. al. ${ }^{136}$ studied the formation and photocatalytic properties of $\mathrm{MoS}_{2} / \mathrm{AlN}(\mathrm{GaN})$ heterostructures. It is found that the constructions of these heterostructures are not energetically costly, while the photocatalytic activity can be greatly improved. As show in Fig. 10(b), the bandgaps and band edge levels of the heterostructures become much closer to the standard requirements for photocatalytic water splitting. Consequently, the light harvesting properties are boosted in ultraviolet-ray and visiblelight wavelengths. What's more, as illustrated in Fig. 10(a), the reduction and oxidation processes will be conducted separately at the opposite sides of the heterostructures during the photocatalytic water splitting, where $\operatorname{AlN}(\mathrm{GaN})$ is an electron donor and $\mathrm{MoS}_{2}$ the electron acceptor, respectively. This will reduce the rate of e-h recombination and further increase the efficiency of the photocatalytic water splitting.

Ideal heterostructures can not only improve the water splitting process, but also enhance the stability of monolayers in solution. It has been theoretically realized that the pure water will not strongly interact with the pristine Phosphorene ${ }^{62}$. However, an exothermic reaction can occur if Phosphorene is first oxidized ${ }^{137}$, which will lead to the degradation of Phosphorene. Constructing the Phosphorene-based heterostructure by a foreign layer as the electron donor can separate the hydrogen and oxygen production processes. Hence, the hydrogen will be produced in the Phosphorene layer and the oxygen on the top of the foreign layer. To achieve such a Phosphorenebased heterostructure, the foreign 2D materials should meet the following conditions: First, a suitable crystal structure inconsistent with the Phosphorene lattice, which is the fundamental to building heterostructures. Slightly larger lattice constants than that of Phosphorene are preferred since Phosphorene is more stable and shows a larger bandgap under the tensile strain than under the compression strain ${ }^{62}$. Secondly, the VBM should be located at the $\Gamma$ point and located slightly higher than that of Phosphorene, which can protect the direct gap nature of Phosphorene and guarantee the oxygen generation process can take place in the foreign layer. At last, the CBM should be placed higher than that of Phosphorene, so that the hydrogen production process can take place on top of the Phosphorene part.

2D materials can also be combined with the surfaces of nanocrystals to form heterogeneous structures. A recent study by Chen et. al. ${ }^{90}$ successfully synthesized $\mathrm{MS}_{2}-\mathrm{CdS}(\mathrm{M}=\mathrm{W}$ or Mo) nanohybrids using a facile one-pot wetchemical method, in which the semiconducting single-layer $\mathrm{MS}_{2}$ with lateral size of 4-10 nm can form on the Cd-rich (0001) surface of wurtzite $\mathrm{CdS}$ nanocrystals. The anionic $\mathrm{S}$ donors $\mathrm{MS}_{4}^{2-}$ selec- 
tively attach to the Cd-terminated (0001) surface and form the nanohybrids, as depicted in Fig. 11. These fabricated structures tend to have many active edge sites in the $\mathrm{MS}_{2}$ layers, which can dramatically improve the the photocatalytic water splitting process (see Fig. 11).

The heterostructure approach is similar to the cocatalyst approach since the working mechanics are much the same. Therefore, the heterostructures containing both semiconducting lattices and metallic lattices may be more efficient than the pure semiconducting heterostructures, considering the metallic lattices can effectively separate the e-h pairs and have more active sites.

(a)

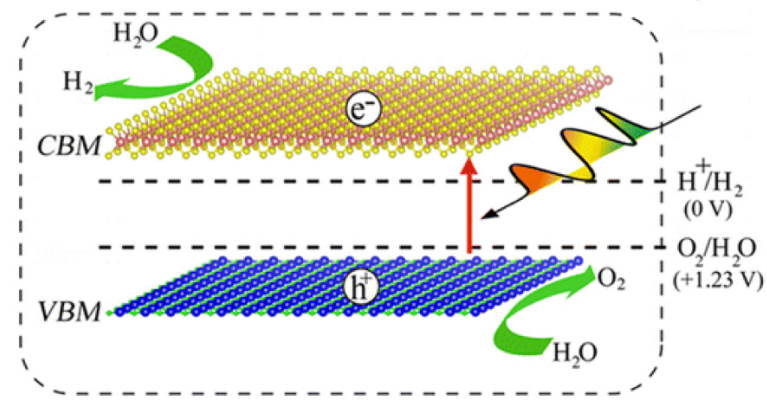

(b)

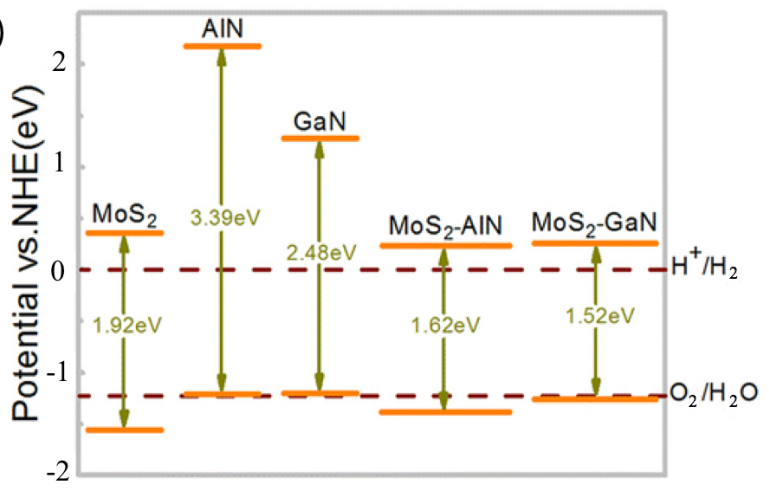

Fig. 10 (a) The structures of $\mathrm{MoS}_{2} / \mathrm{AlN}(\mathrm{GaN})$ heterostructures and (b) their band edge levels. Reproduced with permission of authors in Ref. 136. Copyright 2014 American Chemical Society.

\section{Challenges and Outlook}

From above, we show how the photocatalytic properties can be explored and how DFT simulations can be employed to efficiently screen the photocatalytic potential spaces. Though great progresses have been made, it is still far from the wide application of 2D photocatalysis. There are still many challenges like the low yield/cost efficiency, degradation, kinetics of charge transfer and trap, and recycling. The recycling problem may be resolved by designing suitable devices or tun-
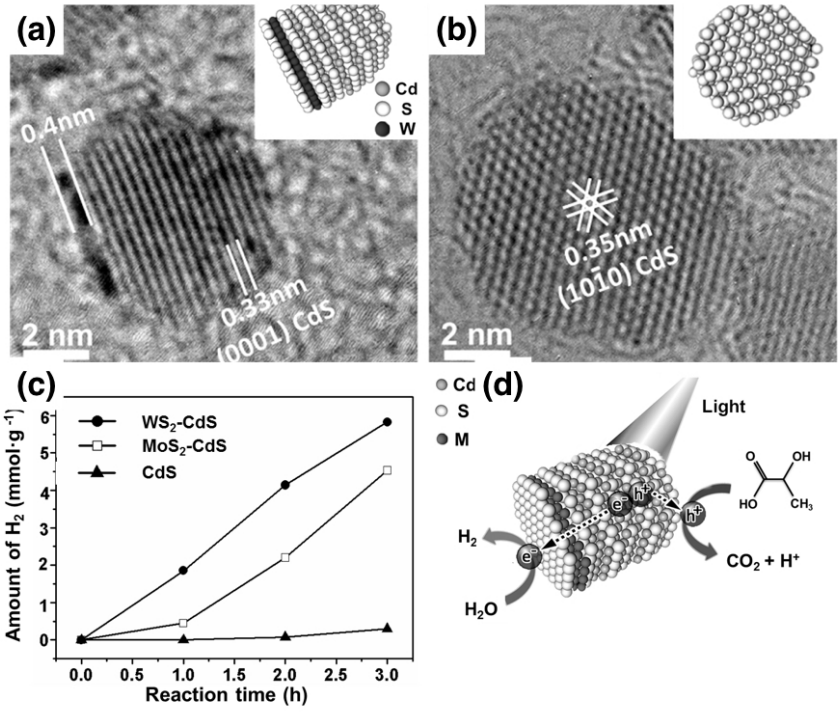

Fig. $11 \mathrm{HR}-\mathrm{TEM}$ images of $\mathrm{WS}_{2}-\mathrm{CdS}$ nanohybrids viewed (a) perpendicularly and (b) parellelly; (c) $\mathrm{H}_{2}$ production for nanohybrids; (d) schematic illustration of the photocatalytic process of nanohybrids in the lactic acid solution. Reproduced with permission of authors in Ref. 90. Copyright 2015 Wiley-VCH Verlag GmbH \& Co. KGaA, Weinheim.

ing magnetic properties, but the efficiency and robustness can only count on the development of more advanced materials. In the theoretical aspect, simulations are efficient for the structure prediction, but not yet proved competent to predict 2D heterojunction structures. The solvation effects are often neglected in current theoretical studies, while such effects are important and the development of accurate solvation model is highly in need. Besides, there is still no general method developed to quantitatively measure the activity of active sites in photocatalytic process. In addition, deeper understanding of the dynamic photocatalytic processes faces challenges in both experiment and theory. For example, some 2D atomic thin materials are often stabilized on substrates. The migration behaviors of the electrons or holes from substrates should have important impacts on the photocatalytic efficiency of 2D photocatalysis, while such effects are not yet fully understood.

However, computer simulations have demonstrated its power in screening and engineering of 2D materials, which has dramatically boosted the development of photocatalysis. The great progress in the field of 2D photocatalysis suggests that many of those challenges can be tackled in the near future.

\section{Acknowledgments}

Y. Li would like to acknowledge the financial support from the NSFC (11674131). Y-L. Li acknowledges the financial 
support from the NSFC (11347007, and 11674131), Qing Lan Project, and the Priority Academic Program Development of Jiangsu Higher Education Institutions (PAPD). B. Sa acknowledges the financial support from the NSFC (61504028). R. Ahuja acknowledges the Swedish Research Council (VR), SSF and STandUP for financial support.

\section{References}

1 A. Fujishima and K. Honda, Nature, 1972, 238, 37-38.

2 A. J. Bard, J. Photochem., 1979, 10, 59-75.

3 A. J. Bard, Science, 1980, 207, 139-144.

4 A. L. Linsebigler, G. Lu and J. T. Yates Jr, Chem. Rev., 1995, 95, 735758.

5 A. Kudo and Y. Miseki, Chem. Soc. Rev., 2009, 38, 253-278.

6 K. Maeda and K. Domen, J. Phys. Chem. Lett., 2010, 1, 2655-2661.

7 A. Kubacka, M. Fernández-García and G. Colón, Chem. Rev., 2011, 112, $1555-1614$.

8 F. E. Osterloh, Chem. Soc. Rev., 2013, 42, 2294-2320.

9 Photoelectrochemical Water Splitting, ed. H.-J. Lewerenz and L. Peter, The Royal Society of Chemistry, 2013, pp. P001-468.

10 T. K. Townsend, Inorganic Metal Oxide Nanocrystal Photocatalysts for Solar Fuel Generation from Water, Springer Science \& Business Media, 2014.

11 K. S. Novoselov, A. K. Geim, S. V. Morozov, D. Jiang, Y. Zhang, S. V. Dubonos, I. V. Grigorieva and A. A. Firsov, Science, 2004, 306, 666669.

12 P. R. Wallace, Phys.Rev., 1947, 71, 622.

13 V. O. Özçelik and S. Ciraci, J. Phys. Chem. C, 2013, 117, 2175-2182.

14 G. Li, Y. Li, H. Liu, Y. Guo, Y. Li and D. Zhu, Chem. Commun., 2010, 46, 3256-3258.

15 D. Malko, C. Neiss, F. Viñes and A. Görling, Phys. Rev. Lett., 2012, 108, 086804.

16 K. Takeda and K. Shiraishi, Phys. Rev. B, 1994, 50, 14916-14922.

17 B. Aufray, A. Kara, S. Vizzini, H. Oughaddou, C. Leandri, B. Ealet and G. Le Lay, Appl. Phys. Lett., 2010, 96, 183102.

18 Z. A. Piazza, H.-S. Hu, W.-L. Li, Y.-F. Zhao, J. Li and L.-S. Wang, Nat. Commun., 2014, 5, 3113.

19 A. Szabo and N. S. Ostlund, Modern quantum chemistry: introduction to advanced electronic structure theory, Courier Corporation, 2012.

20 I. N. Levine, Quantum chemistry, Pearson Higher Ed, 2013.

21 P. Hohenberg and W. Kohn, Phys. Rev., 1964, 136, B864-B871.

22 W. Kohn and L. J. Sham, Phys. Rev., 1965, 140, A1133-A1138.

23 S. M. Woodley and R. Catlow, Nat. Mater, 2008, 7, 937-946.

24 K. Lejaeghere, G. Bihlmayer, T. Björkman, P. Blaha, S. Blügel, V. Blum, D. Caliste, I. E. Castelli, S. J. Clark, A. Dal Corso et al., Science, 2016, 351, 3000

25 A. K. Singh, H. L. Zhuang and R. G. Hennig, Phys. Rev. B, 2014, 89, 245431.

26 S. Zhang, Z. Yan, Y. Li, Z. Chen and H. Zeng, Angew. Chem. Int. Ed. Engl., 2015, 54, 3112-3115.

27 J. Liu, X.-B. Li, D. Wang, H. Liu, P. Peng and L.-M. Liu, J. Mater. Chem. A, 2014, 2, 6755-6761.

28 C. Eames and M. S. Islam, J. Am. Chem. Soc., 2014, 136, 16270-16276.

29 W. Sun, Y. Li, B. Wang, X. Jiang, M. I. Katsnelson, P. A. Korzhavyi, O. Eriksson and I. D. Marco, arXiv:1607.06410, 2016.

30 X.-F. Zhou, X. Dong, A. R. Oganov, Q. Zhu, Y. Tian and H.-T. Wang, Phys. Rev. Lett., 2014, 112, 085502.

31 B. C. Revard, W. W. Tipton, A. Yesypenko and R. G. Hennig, Phys. Rev. $B, 2016,93,054117$.
32 S. J. Singer, J.-L. Kuo, T. K. Hirsch, C. Knight, L. Ojamäe and M. L. Klein, Phys. Rev. Lett., 2005, 94, 135701.

33 Y. Li, C. M. Lousada, I. L. Soroka and P. A. Korzhavyi, Inorg. Chem., 2015, 54, 8969-8977.

34 X. Jiang, P. Wang and J. Zhao, J. Mater. Chem. A, 2015, 3, 7750-7758.

35 P. Wang, X. Jiang and J. Zhao, J. Phys. Condens. Matter, 2015, 28, 034004.

36 L. Xu, Y. Yu, J.-B. Lin, X. Zhou, W. Tian, D. Nieckarz, P. Szabelski and S. Lei, Nanoscale, 2016, 8568-8574.

37 J. P. Perdew and A. Zunger, Phys. Rev. B, 1981, 23, 5048-5079.

38 S. H. Vosko, L. Wilk and M. Nusair, Can. J. Phys., 1980, 58, 1200-1211.

39 J. P. Perdew and Y. Wang, Phys. Rev. B, 1992, 45, 13244-13249.

40 J. Harris and R. Jones, Journal of Physics F: Metal Physics, 1974, 4, 1170.

41 D. C. Langreth and J. P. Perdew, Solid State Commun., 1975, 17, 1425 1429.

42 O. Gunnarsson and B. Lundqvist, Phys. Rev. B, 1976, 13, 4274.

43 J. Harris, International Journal of Quantum Chemistry, 1979, 16, 189193.

44 J. Harris, Phys. Rev. A, 1984, 29, 1648.

45 A. D. Becke, Phys. Rev. A, 1988, 38, 3098-3100.

46 D. C. Langreth and M. J. Mehl, Phys. Rev. B, 1983, 28, 1809-1834.

47 J. Muscat, A. Wander and N. Harrison, Chem. Phys. Lett., 2001, 342, 397-401.

48 J. Heyd, J. E. Peralta, G. E. Scuseria and R. L. Martin, J. Chem. Phys., 2005, 123, 174101.

49 S. Grimme, J. Comput. Chem., 2006, 27, 1787-1799.

50 S. Grimme, WIREs Comput. Mol. Sci., 2011, 1, 211-228.

51 M. Dion, H. Rydberg, E. Schröder, D. C. Langreth and B. I. Lundqvist, Phys. Rev. Lett., 2004, 92, 246401.

52 G. Román-Pérez and J. M. Soler, Phys. Rev. Lett., 2009, 103, 096102.

53 T. Björkman, A. Gulans, A. V. Krasheninnikov and R. M. Nieminen, Phys. Rev. Lett., 2012, 108, 235502.

54 Y. Li, B. Pathak, J. Nisar, Z. Qian and R. Ahuja, EPL (Europhysics Letters), 2013, 103, 28007

55 A. Splendiani, L. Sun, Y. Zhang, T. Li, J. Kim, C.-Y. Chim, G. Galli and F. Wang, Nano letters, 2010, 10, 1271-1275.

56 B. J. Robinson, C. E. Giusca, Y. T. Gonzalez, N. D. Kay, O. Kazakova and O. V. Kolosov, 2D Mater., 2015, 2, 015005.

57 Y. Li, Y.-L. Li, C. M. Araujo, W. Luo and R. Ahuja, Catal. Sci. Technol., 2013, 3, 2214-2220.

58 Y. Liang, S. Huang, R. Soklaski and L. Yang, Applied Physics Letters, 2013, 103, 042106.

59 A. Beal and H. Hughes, J. Phys. C., 1979, 12, 881.

60 A. Molina-Sánchez, M. Palummo, A. Marini and L. Wirtz, Phys. Rev. B, 2016, 93, 155435

61 K. F. Mak, C. Lee, J. Hone, J. Shan and T. F. Heinz, Phys. Rev. Lett., 2010, 105, 136805

62 B. Sa, Y.-L. Li, J. Qi, R. Ahuja and Z. Sun, J. Phys. Chem. C, 2014, 118, 26560-26568.

63 J. P. Perdew, K. Burke and M. Ernzerhof, Phys. Rev. Lett., 1996, 77, 3865-3868.

64 J. Heyd, G. E. Scuseria and M. Ernzerhof, J. Chem. Phys., 2003, 118, $8207-8215$.

65 T. Cheiwchanchamnangij and W. R. Lambrecht, Phys. Rev. B, 2012, 85, 205302.

66 A. H. Reshak and S. Auluck, Phys. Rev. B, 2003, 68, 125101.

67 E. E. Salpeter and H. A. Bethe, Phys. Rev., 1951, 84, 1232.

68 A. Ramasubramaniam, Phys. Rev. B, 2012, 86, 115409.

69 D. Y. Qiu, H. Felipe and S. G. Louie, Phys. Rev. Lett., 2013, 111, 216805.

70 K. Fan, H. Chen, Y. Ji, H. Huang, P. M. Claesson, Q. Daniel, B. Philippe,

14 | Journal Name, 2010, [vol], 1-16 
H. Rensmo, F. Li, Y. Luo et al., Nature Communications, 2016, 7, 11981.

71 J. N. Coleman, M. Lotya, A. O'Neill, S. D. Bergin, P. J. King, U. Khan, K. Young, A. Gaucher, S. De, R. J. Smith, I. V. Shvets, S. K. Arora, G. Stanton, H.-Y. Kim, K. Lee, G. T. Kim, G. S. Duesberg, T. Hallam, J. J. Boland, J. J. Wang, J. F. Donegan, J. C. Grunlan, G. Moriarty, A. Shmeliov, R. J. Nicholls, J. M. Perkins, E. M. Grieveson, K. Theuwissen, D. W. McComb, P. D. Nellist and V. Nicolosi, Science, 2011, 331, 568-571.

72 H. L. Zhuang and R. G. Hennig, Chem. Mater, 2013, 25, 3232-3238.

73 R. Zacharia, H. Ulbricht and T. Hertel, Phys. Rev. B, 2004, 69, 155406.

74 S. Cahangirov, M. Topsakal, E. Aktürk, H. Şahin and S. Ciraci, Phys. Rev. Lett., 2009, 102, 236804.

75 Y. Li, Y.-L. Li, W. Sun and R. Ahuja, Comput. Mater. Sci., 2014, 92, 206-212.

76 Y.-L. Li, Y. Li and C. Tang, Int. J. Hydrogen Energy, 2016.

77 J. Kang, S. Tongay, J. Zhou, J. Li and J. Wu, Appl. Phys. Lett., 2013, 102, 012111.

78 K. F. Mak, M. Y. Sfeir, J. A. Misewich and T. F. Heinz, Proc. Natl. Acad. Sci., 2010, 107, 14999-15004.

79 Z. Y. Zhu, Y. C. Cheng and U. Schwingenschlögl, Phys. Rev. B, 2011, 84, 153402.

80 Y. C. Cheng, Z. Y. Zhu, M. Tahir and U. Schwingenschlögl, EPL, 2013, 102, 57001.

81 D. Xiao, G.-B. Liu, W. Feng, X. Xu and W. Yao, Phys. Rev. Lett., 2012, 108, 196802.

82 H. Zeng, J. Dai, W. Yao, D. Xiao and X. Cui, Nat. Nano., 2012, 7, 490493.

83 K. F. Mak, K. He, J. Shan and T. F. Heinz, Nat. Nano., 2012, 7, 494-498.

84 H. Yuan, M. S. Bahramy, K. Morimoto, S. Wu, K. Nomura, B.-J. Yang, H. Shimotani, R. Suzuki, M. Toh, C. Kloc, X. Xu, R. Arita, N. Nagaosa and Y. Iwasa, Nat. Phys., 2013, 9, 563-569.

85 X. Wang, K. Maeda, A. Thomas, K. Takanabe, G. Xin, J. M. Carlsson, K. Domen and M. Antonietti, Nat. Mater, 2009, 8, 76-80.

86 L. Jia, D.-H. Wang, Y.-X. Huang, A.-W. Xu and H.-Q. Yu, J. Phys. Chem. C, 2011, 115, 11466-11473.

87 U. Maitra, U. Gupta, M. De, R. Datta, A. Govindaraj and C. N. R. Rao, Angew. Chem. Int. Ed. Engl., 2013, 52, 13057-13061.

88 U. Gupta, B. S. Naidu, U. Maitra, A. Singh, S. N. Shirodkar, U. V. Waghmare and C. N. R. Rao, APL Mat., 2014, 2, 092802.

89 B. Mahler, V. Hoepfner, K. Liao and G. A. Ozin, J. Am. Chem. Soc., 2014, 136, 14121-14127.

90 J. Chen, X.-J. Wu, L. Yin, B. Li, X. Hong, Z. Fan, B. Chen, C. Xue and H. Zhang, Angew. Chem. Int. Ed. Engl., 2015, 54, 1210-1214.

91 P. Garg, S. Kumar, I. Choudhuri, A. Mahata and B. Pathak, J. Phys. Chem. C, 2016, 120, 7052-7060.

92 J. Lahiri and M. Batzill, J. Phys. Chem. C, 2008, 112, 4304-4307.

93 J. Yu, C.-Y. Xu, F.-X. Ma, S.-P. Hu, Y.-W. Zhang and L. Zhen, ACS Appl. Mater. Interfaces, 2014, 6, 22370-22377.

94 H. Zhong, G. Yang, H. Song, Q. Liao, H. Cui, P. Shen and C.-X. Wang, J. Phys. Chem. C, 2012, 116, 9319-9326.

95 L. Dou, A. B. Wong, Y. Yu, M. Lai, N. Kornienko, S. W. Eaton, A. Fu, C. G. Bischak, J. Ma, T. Ding et al., Science, 2015, 349, 1518-1521.

96 S. Zhang, M. Xie, F. Li, Z. Yan, Y. Li, E. Kan, W. Liu, Z. Chen and H. Zeng, Angew. Chem. Int. Ed. Engl., 2016, 128, 1698-1701.

97 M. Denk, D. Kuhness, M. Wagner, S. Surnev, F. R. Negreiros, L. Sementa, G. Barcaro, I. Vobornik, A. Fortunelli and F. P. Netzer, ACS Nano, 2014, 8, 3947-3954.

98 J. Liu, X.-B. Li, D. Wang, W.-M. Lau, P. Peng and L.-M. Liu, J. Chem. Phys., 2014, 140, 054707.

99 Z. Lei, W. You, M. Liu, G. Zhou, T. Takata, M. Hara, K. Domen and C. Li, Chem. Commun., 2003, 2142-2143.

100 Z. Xu, Y. Li, S. Peng, G. Lu and S. Li, RSC Adv., 2012, 2, 3458-3466.
101 J. Yu, L. Qi and M. Jaroniec, J. Phys. Chem. C, 2010, 114, 13118-13125.

102 H. De Lasa and B. Serrano-Rosales, Advances in Chemical Engineering: Photocatalytic Technologies, Academic Press, 2009, vol. 36.

103 Z. H. Ni, T. Yu, Y. H. Lu, Y. Y. Wang, Y. P. Feng and Z. X. Shen, ACS Nano, 2008, 2, 2301-2305.

104 R. Fei and L. Yang, Nano Lett., 2014, 14, 2884-2889.

105 S. Rodin, A. A. Carvalho and H. Castro Neto, A. Phys. Rev. Lett., 2014, 112, 176801

106 C. Lee, X. Wei, J. W. Kysar and J. Hone, Science, 2008, 321, 385-388.

107 Q. Wei and X. Peng, Appl. Phys. Lett., 2014, 104, -.

108 M. Elahi, K. Khaliji, S. M. Tabatabaei, M. Pourfath and R. Asgari, Phys. Rev. B, 2015, 91, 115412.

109 X. Peng, Q. Wei and A. Copple, Phys. Rev. B, 2014, 90, 085402.

110 G. Plechinger, A. Castellanos-Gomez, M. Buscema, H. S. J. van der Zant, G. A. Steele, A. Kuc, T. Heine, C. Schüller and T. Korn, 2D Mater, 2015, 2, 015006.

111 S. Bertolazzi, J. Brivio and A. Kis, ACS nano, 2011, 5, 9703-9709.

112 X. Qin, D. Yang, R. Frindt and J. Irwin, Ultramicroscopy, 1992, 42-44, Part 1, 630 - 636 .

113 M. P. Álvarez, E. del Corro, Á. Morales-García, L. Kavan, M. Kalbac and O. Frank, Nano Lett., 2015, 15, 3139-3146.

114 D. W. Hwang, H. G. Kim, J. S. Lee, J. Kim, W. Li and S. H. Oh, J. Phys. Chem. B, 2005, 109, 2093-2102.

115 Z. Guo, B. Sa, B. Pathak, J. Zhou, R. Ahuja and Z. Sun, Int. J. Hydrogen Energy, 2014, 39, 2042 - 2048.

116 X. Zou, M. Liu, Z. Shi and B. I. Yakobson, Nano Lett., 0, 0, 3495-3500.

117 Y. Sun, S. Gao, F. Lei and Y. Xie, Chem. Soc. Rev., 2015, 44, 623-636.

118 C. Ataca and S. Ciraci, Phys. Rev. B, 2012, 85, 195410.

119 Q. Tay, P. Kanhere, C. F. Ng, S. Chen, S. Chakraborty, A. C. H. Huan, T. C. Sum, R. Ahuja and Z. Chen, Chem. Mater, 2015, 27, 4930-4933.

120 A. Kuc and T. Heine, Chem. Soc. Rev., 2015, 44, 2603-2614.

121 A. J. Nozik and R. Memming, J. Phys. Chem. Lett., 1996, 100, 1306113078.

122 J. Yang, D. Wang, H. Han and C. Li, Acc. Chem. Res., 2013, 46, 19001909.

123 P. V. Kamat, J. Phys. Chem. Lett., 2012, 3, 663-672.

124 W. Wang, X. Huang, S. Wu, Y. Zhou, L. Wang, H. Shi, Y. Liang and B. Zou, Appl. Catal., B., 2013, 134, 293-301.

125 X. Lin, J. Xing, W. Wang, Z. Shan, F. Xu and F. Huang, J. Phys. Chem. C, 2007, 111, 18288-18293.

126 Z. Jiang, Y. Liu, M. Li, T. Jing, B. Huang, X. Zhang, X. Qin and Y. Dai, Sci. Rep., 2016, 6, 22727.

127 P. Kulis, J. Butikova, B. Polyakov, G. Marcins, J. Pervenecka, K. Pudzs and I. Tale, IOP CONF., 2012, p. 012048.

128 U. Bhanu, M. R. Islam, L. Tetard and S. I. Khondaker, Sci. Rep., 2014, 4, 5575 .

129 A. Bumajdad and M. Madkour, Phys. Chem. Chem. Phys., 2014, 16, 7146-7158.

130 K. Awazu, M. Fujimaki, C. Rockstuhl, J. Tominaga, H. Murakami, Y. Ohki, N. Yoshida and T. Watanabe, J. Am. Chem. Soc., 2008, 130, 1676-1680.

131 F. Wang, P. Stepanov, M. Gray and C. N. Lau, Nanotechnology, 2015, 26, 105709.

132 Y. Sun, H. Liu, F. Zhou, L. Yang, S. He, B. Sun and J. Liu, Chem. Eur. $J, 2014,20,10414-10424$.

133 Z. Qian, B. Pathak, J. Nisar and R. Ahuja, J. Nanopart. Res., 2012, 14, $1-7$.

134 Y. Shiga, N. Umezawa, N. Srinivasan, S. Koyasu, E. Sakai and M. Miyauchi, Chem. Commun., 2016, 52, 7470-7473.

135 J. Linghu, C. Zhang, Y. P. Feng, L. Shen et al., Appl. Phys. Lett., 2016, 108, 122105 .

136 J. Liao, B. Sa, J. Zhou, R. Ahuja and Z. Sun, J. Phys. Chem. C, 2014, 
118, 17594-17599.

137 G. Wang, W. J. Slough, R. Pandey and S. P. Karna, 2D Mater, 2016, 3, 025011 . 